\title{
Clinical communication patterns of veterinary practitioners during dairy herd health and production management farm visits
}

\author{
Caroline Ritter, ${ }^{11}$ Cindy L. Adams, $†$ David F. Kelton, $\ddagger$ and Herman W. Barkema* \\ *Department of Production Animal Health, and \\ †Department of Veterinary Clinical and Diagnostic Sciences, Faculty of Veterinary Medicine, University of Calgary, 2500 University Dr., Calgary, \\ AB, T2N 1N4, Canada \\ ‡Department of Population Medicine, University of Guelph, 50 Stone Rd., Guelph, ON, N1G 2W1, Canada
}

\begin{abstract}
Effective communication with clients is an important skill for veterinary practitioners and is linked to clients' satisfaction with the consultancy and adherence to medical advice. Detailed description of veterinary communication styles in companion animal contexts has added to the communication knowledge base and informed veterinary curricula and postgraduate education programs. The objective here was to describe veterinary practitioners' communication patterns during dairy herd health and production management farm visits. Fourteen veterinarians in Alberta and Ontario, Canada, were provided with action cameras (Hero3 black edition; GoPro Inc., San Mateo, CA) and recorded 3 to 7 farm visits each. The resulting 70 audio-video recordings were analyzed using the Roter Interaction Analysis System. Additionally, demographic variables were obtained from study participants to investigate associations of these variables with communication patterns. Intraclass correlation coefficients were calculated for communication units, and multilevel negative binomial regression was used to compare communication patterns between demographic groups. Additionally, the relationship-centeredness of interactions was evaluated and compared between groups using linear regression models. Communication patterns varied considerably among recordings of the same veterinarian interacting with different clients. However, most veterinary talk focused on farmer education, counseling, and building a relationship, whereas especially open-ended questions were rare. When discussion revolved around health issues of an individual animal, veterinarians used less social talk but focused more on biomedical information gathering. Veterinarians' age and sex and the length of the veterinarian-farmer relationship had limited association with communications; however, if the farmer
\end{abstract}

Received March 14, 2018

Accepted July 9, 2018.

${ }^{1}$ Corresponding author: cmnritte@ucalgary.ca and veterinarian were both male, the conversation was more relationship centered. Communication of veterinarians with previous communication training was very similar to that of those without training. Detailed description of veterinary practitioners' communication patterns on dairy farms will contribute to establishing the importance of communication as a clinical skill and is critical to identifying unique aspects of veterinary on-farm communication.

Key words: veterinary communication, dairy farm consultancy, on-farm video recording, Roter interaction analysis system

\section{INTRODUCTION}

Effective communication is crucial for veterinary practitioners; veterinarians and their clients often regard communication as important or as more important than clinical knowledge (Mellanby et al., 2011; McDermott et al., 2015). Based on an extensive body of research, developed over decades in human medicine (described by Silverman et al., 2013) and more recently in companion animal medicine (described by Adams and Kurtz, 2017), improving clinical communication in specific ways leads to more effective clinical consultations. In particular, relationship-centered communication, defined by a balance of power between the veterinarian and farmer and including inquiries about social aspects of the presented problem (e.g., how does an animal's sickness affect the owner's daily routine), has been demonstrated to improve the accuracy of information gathering, client satisfaction, and adherence to advice through an improved relationship (Stewart et al., 2003; Kanji et al., 2012; McArthur and Fitzgerald, 2013).

Although the research is compelling, the majority of studies assessing communication patterns between veterinarian and client have been conducted in companion animal settings (e.g., Shaw et al., 2006; Coe et al., 2009; Nogueira Borden et al., 2010; Mossop et al., 2015). For example, veterinary communication patterns often dif- 
fered depending on the reason for a pet owner's visit. There was more social talk and more rapport building during wellness appointments (i.e., consultations to prevent future problems such as vaccination appointments), whereas veterinarians had an increased emphasis on gathering biomedical content during health problem consultations (i.e., appointments to address a specific problem; Shaw et al., 2008). The authors concluded that this focus on biomedical information might cause veterinarians to oversee potential social concerns or concerns related to the animal's lifestyle such as diet or exercise regimen, which could affect client satisfaction and adherence.

Veterinary educators in many countries acknowledge the importance of clinical communication skills by including findings from these studies (and many others) in veterinary communication training. However, although most research exploring veterinary communication was conducted in Europe, there are strong indicators that veterinary communication in the food animal setting needs improvement (Cipolla and Zecconi, 2015). Food animal practitioners frequently had a reactive role instead of being a proactive farm advisor, were often unable to identify a dairy farmer's main farm goal, or had different priorities than the farmer (Mee, 2007; Lam et al., 2011; Derks et al., 2013). Additionally, 3 European studies described cattle practitioners' communication patterns evaluating 15 to 30 interactions, and concluded that veterinarians rarely set an agenda, discussed farmers' needs, or elicited farmers' opinions (Jansen et al., 2010; Derks et al., 2013: Bard et al., 2017). However, apparently no study has ever described clinical communication on farms in as much detail as research from companion animal clinics.

Objectives of this study were to (1) describe veterinary communication patterns of herd health and production management (HH\&PM) farm visits; (2) investigate associations of veterinary communication patterns with factors such as veterinarian age and sex, duration of the professional work relationship between veterinarian and farmer, previous clinical communication training, and nature of the conversation (i.e., whether veterinarians communicated differently when problems related to an individual animal were discussed compared with general herd consultancy); and (3) describe the relationshipcenteredness of the interaction and its association with veterinarian and farmer demographics.

\section{MATERIALS AND METHODS}

\section{Participant Recruitment and Collection of Recordings}

Between October 2015 and January 2017, a list of all dairy veterinarians who had at least 10 dairy cli- ents and practiced within a feasible driving distance $(<750 \mathrm{~km})$ from Calgary, Canada, was created based on the authors' familiarity with practicing veterinarians in Alberta and the database of the Alberta Johne's Disease Initiative (Wolf et al., 2015), which included most Alberta dairy practitioners with $>10$ dairy clients. Then, listed veterinarians were contacted by email and telephone and asked to enroll in the study. Of the 16 contacted veterinarians, 11 (69\%) agreed to participate. Reasons for veterinarians to refuse participation included lack of time and feeling uncomfortable with recording HH\&PM visits. Additionally, 30 dairy practitioners who participated in the Dairy Health Management Continuing Education Program (Ontario Veterinary College, University of Guelph, Guelph, ON, Canada) were asked to participate in the study by sending out a group email. Six of these veterinarians (5 from Ontario and 1 from Alberta) agreed to participate in the study from June 2017 to September 2017. Veterinarians were informed that they would receive Can $\$ 100$ if they completed all study aspects.

Ethics approval was obtained from the University of Calgary's Research Ethics board (REB15-1800). Veterinarians who participated in the study obtained written consent from their dairy clients to record video and audio during regular HH\&PM farm visits. Information about the study and contact information for researchers were provided to veterinarians to be distributed to farmers. Each veterinarian was asked to record 7 HH\&PM visits on different farms; however, in 2 cases, the veterinarians did not have enough dairy clients who were willing to be recorded to reach that number, and in 2 other cases, the veterinarians stopped recording farm visits before reaching 7 recordings. The self-reported reason for ceasing participation was the time required to prepare the study equipment. In total, 99 recordings were submitted. Of these, recordings that were deemed insufficient for analysis (i.e., poor audio quality, large parts of the HH\&PM farm visit were not recorded; $\mathrm{n}=18)$, had missing paperwork $(\mathrm{n}=7)$, or for which farmers withdrew their consent after recording $(\mathrm{n}=2)$ were discarded. If fewer than 3 recordings suitable for analysis remained for any particular veterinarian, none of his or her recordings were used. The rationale was that in previous studies, communication patterns varied considerably among visits of the same veterinarian (Shaw et al., 2006; Ritter et al., 2018), and we believed that analyzing fewer than 3 recordings increased the risk of nonrepresentative data. Therefore, 70 recordings by 14 veterinarians $(3-7$ recordings per veterinarian) were analyzed. Farmers provided information on their demographics (e.g., age, sex) by returning a survey that was given to them by the veterinarian in a sealed and stamped envelope addressed to the first 
author. Veterinarians were asked to complete a survey (including demographic factors) emailed to them after they had completed their recordings.

\section{Equipment}

Equipment used for this study has been described in detail (Ritter et al., 2018). In short, veterinarians were provided with a GoPro camera (Hero3 black edition; GoPro Inc., San Mateo, CA) and a BacPac battery (GoPro Inc.), which increased the maximum recording time to approximately $3 \mathrm{~h}$. The camera could be worn on the veterinarian's chest or head using adjustable mounts. A new SD memory card was used for every HH\&PM farm recording. Veterinarians handed a voice recorder to the farmer to wear during the herd visit, ensuring that the farmer's voice was captured even when distances between veterinarian and farmer were too large for the GoPro camera to record the farmer's audio. Files from the camera and the voice recorder were subsequently merged (Ritter et al., 2018).

\section{Coding of Recordings}

The most widely used analytical tool to obtain comprehensive information on communication in a clinical context is the Roter interaction analysis system (RIAS; Roter and Larson, 2002). The RIAS was developed to assess medical encounters in human medicine, and the majority of studies have used the RIAS in this context (reviewed by Pires and Cavaco, 2014). However, suitability of this tool for veterinarian-farmer interactions during dairy farm visits was recently demonstrated (Ritter et al., 2018). Recordings were assessed by trained coders at Johns Hopkins Bloomberg School of Public Health using the RIAS (Roter and Larson, 2002; Shaw et al., 2004). The first and second authors met with the coders before the coding process to familiarize them with the dairy context and ensure appropriate adaptation of codes. Veterinarians did not receive any feedback on their communication during the course of the study.

Using the RIAS, one of 49 mutually exclusive and exhaustive codes was assigned to the smallest unit of statement or expression that constituted a complete thought (i.e., a communication unit) from the veterinarian. For example, a veterinarian's communication unit "You are right" would be coded as an agreement statement, whereas "I believe it will be okay" would be coded as a reassurance statement (Table 1). Although coding criteria were identical, to better reflect the dairy context we decided to change the RIAS code expression "lifestyle" to "animal care," which covered general management topics such as animal nutrition or housing. For example, the veterinarian's communication unit "How do you like the new feed?" would be an animal care open-ended question. In contrast, the RIAS code "biomedical" represented questions or statements related to

Table 1. Examples of veterinarians' communication units and their corresponding Roter interaction analysis system coding (modified from Shaw et al., 2004; Ritter et al., 2018)

\begin{tabular}{|c|c|}
\hline Components and variables ${ }^{1}$ & Examples of veterinarian's communication unit \\
\hline \multicolumn{2}{|l|}{ Information gathering } \\
\hline Open-ended questions & What is on the agenda today? \\
\hline Closed-ended questions & Did you breed this cow already? \\
\hline \multicolumn{2}{|l|}{ Education and counseling } \\
\hline Information giving and counseling & You should move her to a different pen. \\
\hline \multicolumn{2}{|l|}{ Relationship building } \\
\hline \multicolumn{2}{|l|}{ Facilitation and activation } \\
\hline Checks for understanding & Did I get that right? \\
\hline Asks for service & Could you hand me the bucket, please? \\
\hline \multicolumn{2}{|l|}{ Rapport building } \\
\hline Social talk & My kids are starting school this summer \\
\hline Agreement & You are right. \\
\hline Reassurance/optimism & I am sure it will be okay. \\
\hline Concern/worry & I am worried about your conception rate. \\
\hline Approval & It was great that you cleaned the pens. \\
\hline Self-disclosure & I don't like Jersey cows. \\
\hline Empathy & I see how this must be worrying for you. \\
\hline \multicolumn{2}{|l|}{ Negative talk } \\
\hline Disapproval & I don't think this is right. \\
\hline Criticism & He was not very helpful. \\
\hline \multicolumn{2}{|l|}{ Procedural statements } \\
\hline Transition & Now that we looked at the calves, let's go to the heifers. \\
\hline Orientation & I will get the vaccines. \\
\hline
\end{tabular}


specific animal health issues (e.g., "Have you started the treatment yet?").

Frequency of each code during the recordings was counted to create a quantitative data set (Roter and Larson, 2002). Codes were also combined into categories (i.e., communication components) such as "information gathering," "education and counseling," and "relationship building" to reflect the broader form and content of the conversation (Table 1).

In addition to verbal codes describing conversations in detail, RIAS coders assigned global affect ratings after each on-farm recording on a 6 -point Likert scale (1 and 6 representing the lowest and highest possible scores, respectively) reflecting their perception of the veterinarian's affective factors such as warmth, respectfulness, and dominance. Perceptions of the coders were based on communication content (i.e., what the veterinarian said) as well as tone of voice and body language (i.e., nonverbal communication).

To compare whether veterinarians communicated differently depending on the context, specific criteria were used to distinguish sequences within the HH\&PM visit that were categorized as discussions regarding issues with a specific animal compared with animal herd health conversations. In the former case, either the veterinarian or the farmer had to believe that there was a problem with an individual animal. Indicators that were used by the RIAS coders to identify these health problem discussions included verbalizing a concern about an animal, separation of the animal from the group, and use of diagnostic tools (e.g., stethoscope, thermometer), application or recommended application of pharmaceuticals, veterinarian diagnosis. Use of ultrasonography to assess pregnancy or fertility status and routine procedures such as dehorning calves were not classified as health problem conversations unless a concern about a specific animal was mentioned. Previous clinical communication training was defined as having had at least $20 \mathrm{~h}$ of professional communication training, including at least 1 official assessment of communication competence (e.g., through objective structured clinical examinations).

Similar to previous studies in human medicine, the relationship-centeredness care (RCC) score of the onfarm interaction was calculated; in human medicine this is referred to as patient-centeredness (Weiner et al., 2013). The RCC score was obtained by dividing the number of communication units that represent RIAS variables contributing to a good relationship, farmer empowerment, veterinarian's understanding of the whole-farming enterprise, and shared decision making (e.g., veterinarian's questions about animal care topics; statements of empathy, reassurance, and partnership; farmer questions) by the number of RIAS communication units that represent veterinary dominance and a focus on biomedical topics (e.g., medical questions, education from the veterinarian and farmer medical information sharing). Hence, the higher the RCC score, the higher the relationship-centeredness of the farm visit.

\section{Statistical Analyses}

Stata IC15.0 (StataCorp LP, College Station, TX) was used for statistical analysis and $P<0.05$ was considered significant. Descriptive statistics were estimated on demographic variables for veterinarians and farmers as well as on recorded communication units and components. In general, communication patterns were reported for the entire recording as a whole, and percentages of a specific communication unit were obtained by dividing it by the total number of veterinary statements. However, for the purpose of comparing conversations concerning an individual animal and whole-herd discussions, communication units were stratified accordingly. Hence, to obtain the percentage of a specific communication unit, counts of that communication unit were divided by total number of statements made during the whole-herd advisory part and individual-animal discussions, respectively.

Number of communication units was compared among groups. When a comparison was made using demographic characteristics measured on a discrete scale, stratification was based on the median. Then, negative binomial regression models with veterinarian as random effect and length of recording as offset were applied. Hence, groups were compared regarding their number of statements for each minute of recording. Recording length was reported and compared between groups using multilevel linear regression, with veterinarian as random effect.

Intraclass Correlation. To assess whether variance of observed communication patterns or global affect ratings was due to variability among recordings by the same veterinarian or to variability among veterinarians, intraclass correlation coefficients (ICC) were calculated using multilevel linear regression models, with veterinarian as random effect. To account for varying lengths of submitted recordings and standardize the number of recorded communication units, recording length and number of total veterinary communication units were considered denominators.

$\boldsymbol{R} \boldsymbol{C C}$ Score. Because the ICC of the RCC score was very close to zero, linear regression models with clustered random errors for veterinarian were applied to compare RCC scores among groups. Univariable 
correlations between demographic variables and $\mathrm{RCC}$ score were described using categorical explanatory variables as well as using the actual numbers for variables measured on a discrete scale (e.g., years, number of cows). Because the study included only 7 female farmers, a comparison depending on farmer sex was not done. However, effects of sex dyads were described by comparing farms visits where both farmer and veterinarian were male (i.e., male concordance) with farm visits where at least 1 of them was female.

Variables with $P \leq 0.30$ in the univariable regression analysis with RCC score as dependent variable (using discrete scale for explanatory variables, where applicable) were entered into a multivariable linear regression model, with clustered random errors for veterinarian. Interaction terms were considered based on plausibility and if both variables were $P \leq 0.30$ in univariable analysis. Then, backward elimination was done using Bayesian information criterion estimate to assess model fit and obtain the final model. All variables with $P \leq$ 0.05 were included in the final model.

Intrarater Reliability. Intrarater reliability for RIAS codes was assessed by recoding 7 interactions and calculating the Pearson correlation coefficient for categories that had a mean communication unit frequency of at least 1 across all interactions. To evaluate intrarater reliability for global affect ratings, the same
Likert scale was applied, and percentage of agreement for each category was calculated.

\section{RESULTS}

\section{Demographic Factors of Study Participants}

Ten male and 4 female veterinarians participated in the study, providing 46 and 24 recordings, respectively (Table 2). Veterinarians were on average $42 \mathrm{yr}$ old, had an average of $14 \mathrm{yr}$ of dairy herd health experience, and conducted 28 HH\&PM farm visits per month. Participating veterinarians were educated at 3 Canadian veterinary schools. Of the 14 veterinarians, 8 stated that they had extensive communication education during their veterinary education (i.e., all $4 \mathrm{yr}$ ) and had been examined on the communication material. Five veterinarians did not have any communication training, whereas 1 veterinarian had participated in courses during conferences but not during veterinary education.

Farmers (63 men and 7 women) were on average 39 yr old and had 125 lactating cows with an average milk production of $35 \mathrm{~kg} /$ cow per day. They had on average $15 \mathrm{yr}$ of dairy herd health management experience, 1.7 HH\&PM farm visits per month, and had worked with their current veterinarian for 8 yr (Table 2).

Table 2. Demographic characteristics of veterinarians and dairy farmers participating in the study

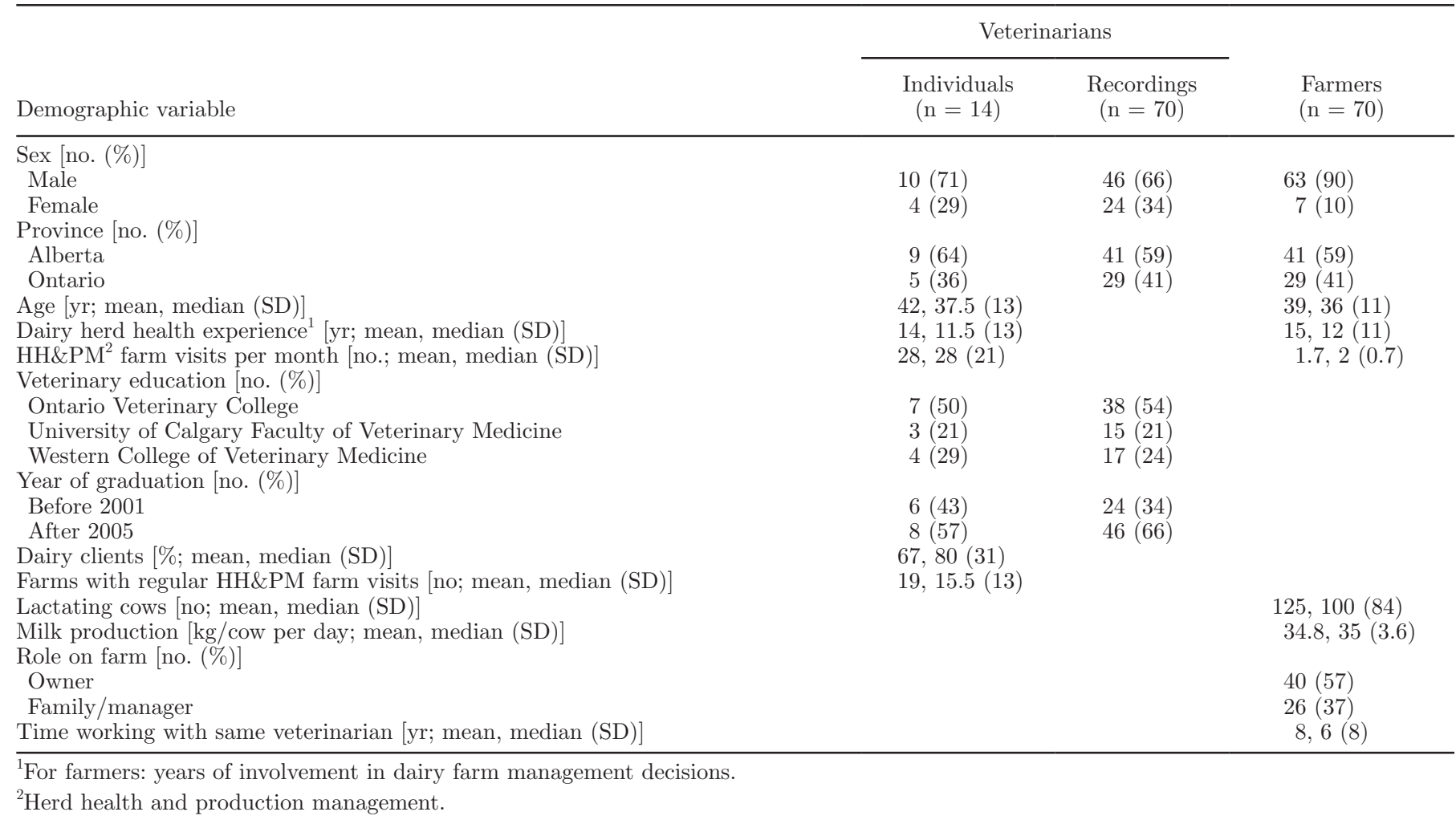


Table 3. Global ratings for veterinarians' affective dimensions during herd health and production management dairy farm visits (measured on 6-point Likert scales)

\begin{tabular}{lcccc}
\hline Affective dimension & Mean & Median & Range & ICC $^{1}(95 \%$ CI) \\
\hline Anxiety/nervousness & 1.2 & 1 & $1-3$ & $0.29(0.10$ to 0.60$)$ \\
Dominance & 3.5 & 3 & $2-5$ & $0.46(0.22$ to 0.71$)$ \\
Interest/attentiveness & 4.1 & 4 & $2-5$ & $0.27(0.09$ to 0.60$)$ \\
Warmth & 4.2 & 4 & $2-5$ & $0.17(0.03$ to 0.57$)$ \\
Responsiveness/engagement & 4.3 & 5 & $2-5$ & $0.19(0.05$ to 0.52$)$ \\
Sympathetic/empathetic & 3.9 & 4 & $2-5$ & $0.33(0.12$ to 0.62$)$ \\
Hurried/rushed & 2.8 & 3 & $1-5$ & $0.42(0.19$ to 0.69$)$ \\
Respectfulness & 3.9 & 4 & $2-5$ & $0.27(0.09$ to 0.60$)$ \\
\hline
\end{tabular}

${ }^{1}$ Intraclass correlation coefficient.

\section{Communication Results}

Average recording length was 82 min (range: $26-172$ min). On average, 10 veterinary statements per recording ( $2 \%$ of the total veterinary talk) were not understandable for the coders and hence were coded as unintelligible communication units. Intrarater reliability was very high, with an overall correlation on veterinary RIAS categories of 0.99 and all veterinary global affect ratings having an agreement of $100 \%$.

Global Affect Ratings. Overall, coders' perceptions of veterinarians' affective dimensions (Table 3) revealed that veterinarians did not appear anxious or nervous (mean: 1.2; median: 1). Their responsiveness and engagement received the highest affect rating score (mean: 4.3; median: 5). However, the range of the ratings was generally wide, encompassing the majority of possible scores. The ICC for global ratings was $\leq 0.50$, ranging from 0.17 (warmth) to 0.46 (dominance), indicating that veterinarians' affective dimensions varied considerably among HH\&PM farm visits.

Communication Units. Veterinarians spent $7 \%$ of their total talk on information gathering (Table 4). Questions were either directly related to the animals' health (i.e., biomedical questions), such as questions about specific health issues, sickness signs, and treatment, or related to animal care factors such as animal nutrition or housing management. Overall, closedended questions about biomedical topics dominated the information-gathering component, with open-ended questions being far less frequent. Farmer education and counseling accounted for $41 \%$ of all veterinary communication units, with considerably more statements related to biomedical topics (including statements regarding pregnancy or fertility status) compared with animal care topics. The component of veterinary relationship building accounted for $44 \%$ of all veterinary communication units and consisted of farmer activation and facilitation ( $8 \%$ of all talk; e.g., veterinarian asks for service or checks for correct understanding); rapport building ( $36 \%$ of all talk; e.g., social talk, expres- sion of agreement, laughter, or approval); and negative talk ( $<1 \%$ of all talk), which included veterinary criticism and disagreement statements. Finally, procedural statements (i.e., transition and orientation statements) accounted for $5 \%$ of veterinary talk.

The ICC for the individual communication units and components differed considerably depending on whether they were standardized by time or by number of all veterinary statements (Table 4). However, similar to the global affect ratings, estimated ICC were generally $\leq 0.50$, indicating that the observed variance in number of communication units was mainly due to variations among recordings of the same veterinarian and less to variations among recordings of different veterinarians.

Sex of Veterinarian. Comparisons between male and female veterinarians did not reveal differences regarding communication patterns and recording length, with the exception that male veterinarians asked more closed-ended animal care questions (10 vs. 6 questions for male and female veterinarians, respectively; $P=$ $0.04)$.

Veterinarian Age and Professional Relationship with the Farmer. Veterinarians $>37$ yr of age educated and counseled farmers more on animal care topics $(P=0.04)$ and used more self-disclosure statements $(P=0.01)$ compared with younger veterinarians (Table 5). Furthermore, veterinarians who had a longer professional relationship with their farmer $(>5$ yr) asked fewer questions (especially fewer biomedical closed-ended questions; $P=0.01$ ) and expressed less reassurance or optimism $(P=0.02)$, and their recordings were longer $(P=0.03$; Table 5$)$.

Communication Training. Previous communication training was positively associated with veterinary use of facilitation and activation statements $(P=0.02)$ but was negatively correlated with number of procedural statements $(P=0.03$; Table 6$)$.

Herd Health and Individual Animal Discussions. Comparison of veterinary communication patterns between whole-herd discussions and individualanimal conversations revealed several differences (Table 
7). Specifically, herd advisory included more animal care-related questions (especially closed-ended questions; $P<0.01)$, animal care-related education and counseling $(P<0.001)$, and more statements related to building a relationship $(P<0.001)$. This difference was mainly due to more social talk $(P<0.001)$ and laughter $(P<0.001)$ during the herd advisory part. In contrast, conversations related to a specific animal were characterized by more biomedical education and counseling statements $(P<0.001)$, more concern $(P$ $<0.001)$, and more empathy statements $(P=0.01)$ expressed by the veterinarian.

$\boldsymbol{R} C \boldsymbol{C}$ Score. There was a negative univariable association of the RCC score with number of transrectal pregnancy or fertility examinations $(P=0.04$; Table 8$)$ and recording length $(P=0.01)$ when regression analysis was conducted with discrete explanatory variables. Variables included in the final multivariable regression model with a significant positive relationship with RCC score were the veterinarian practicing in Ontario compared with Alberta $(P=0.01)$, veterinarian and farmer both being male compared with having a female veterinarian or farmer $(P=0.01)$, and higher average milk production $(P=0.02$; Table 9$)$. Recording length remained negatively correlated with RCC score $(P<$ $0.001)$.

\section{DISCUSSION}

This study is the first to describe dairy practitioners' communication patterns during dairy farm visits using the RIAS. As such, similar to previous studies (e.g., Lam et al., 2011; Derks et al., 2013), it recognized the importance of relationships between veterinarians and their clients that move beyond a strictly medical focus. It is, therefore, another step toward acknowledgment of the importance of communication skills to facilitate improved animal well-being through a better understanding of human aspects that are an essential part of animal care (Williams and Jewell, 2012).

Demographic characteristics of the study population were comparable with the results in other studies regarding veterinarian and dairy farmer age (Winder et al., 2016), duration of veterinarian experience in prac-

Table 4. Selection of communication patterns and intraclass correlation coefficients (ICC) of 14 dairy practitioners during 70 herd health and production management farm visits ${ }^{1}$

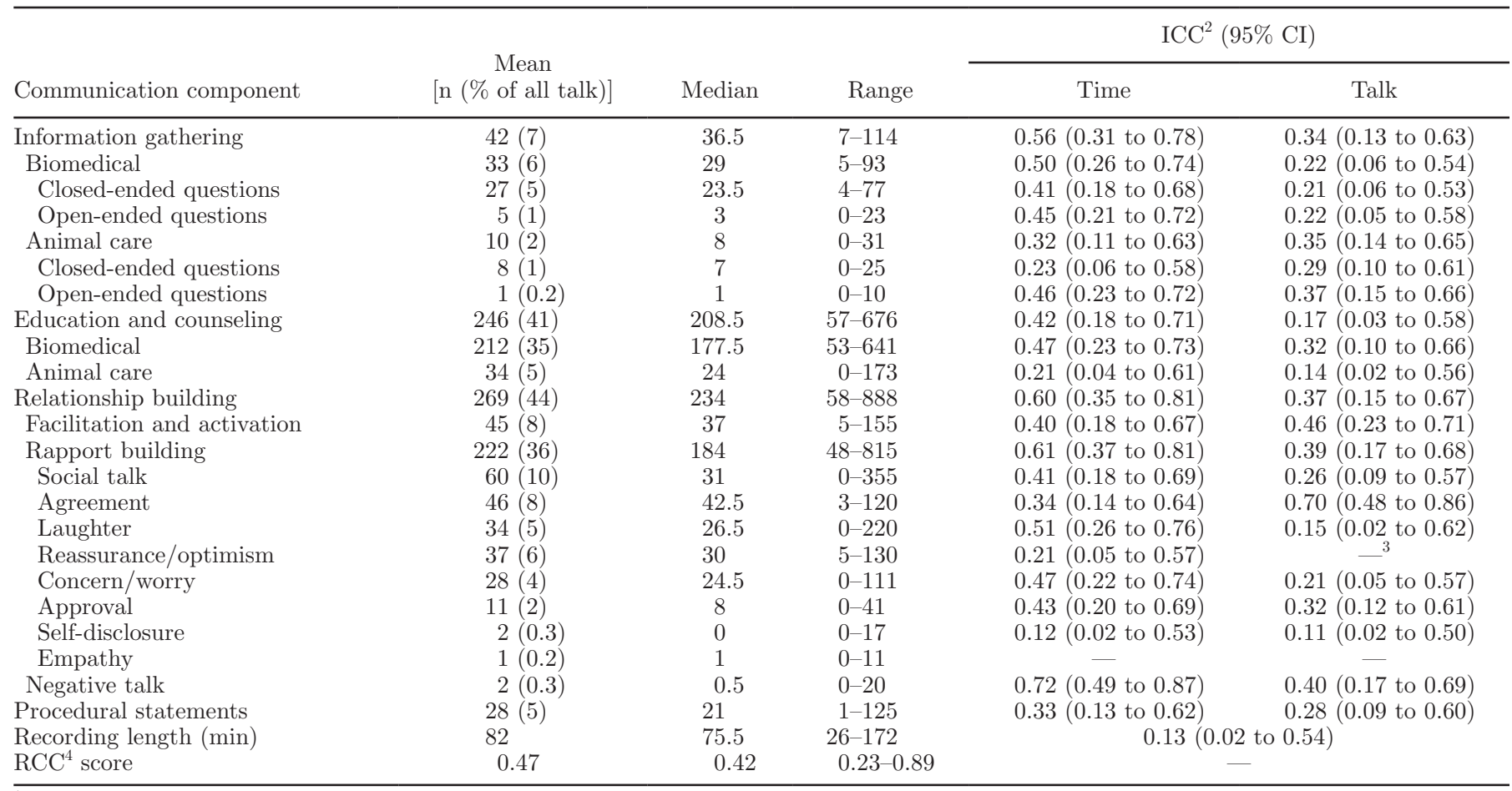

${ }^{1}$ Not displayed are communication units (contributing a total of $3 \%$ to all veterinary talk) that were extremely rare or deemed irrelevant for this study (e.g., unintelligible utterances, bid for repetition).

${ }^{2}$ Standardized by time or by talk.

${ }^{3}$ Dash indicates inability to obtain ICC and $95 \%$ CI due to within-veterinarian correlation very close to 0 .

${ }^{4}$ Relationship-centered care. 


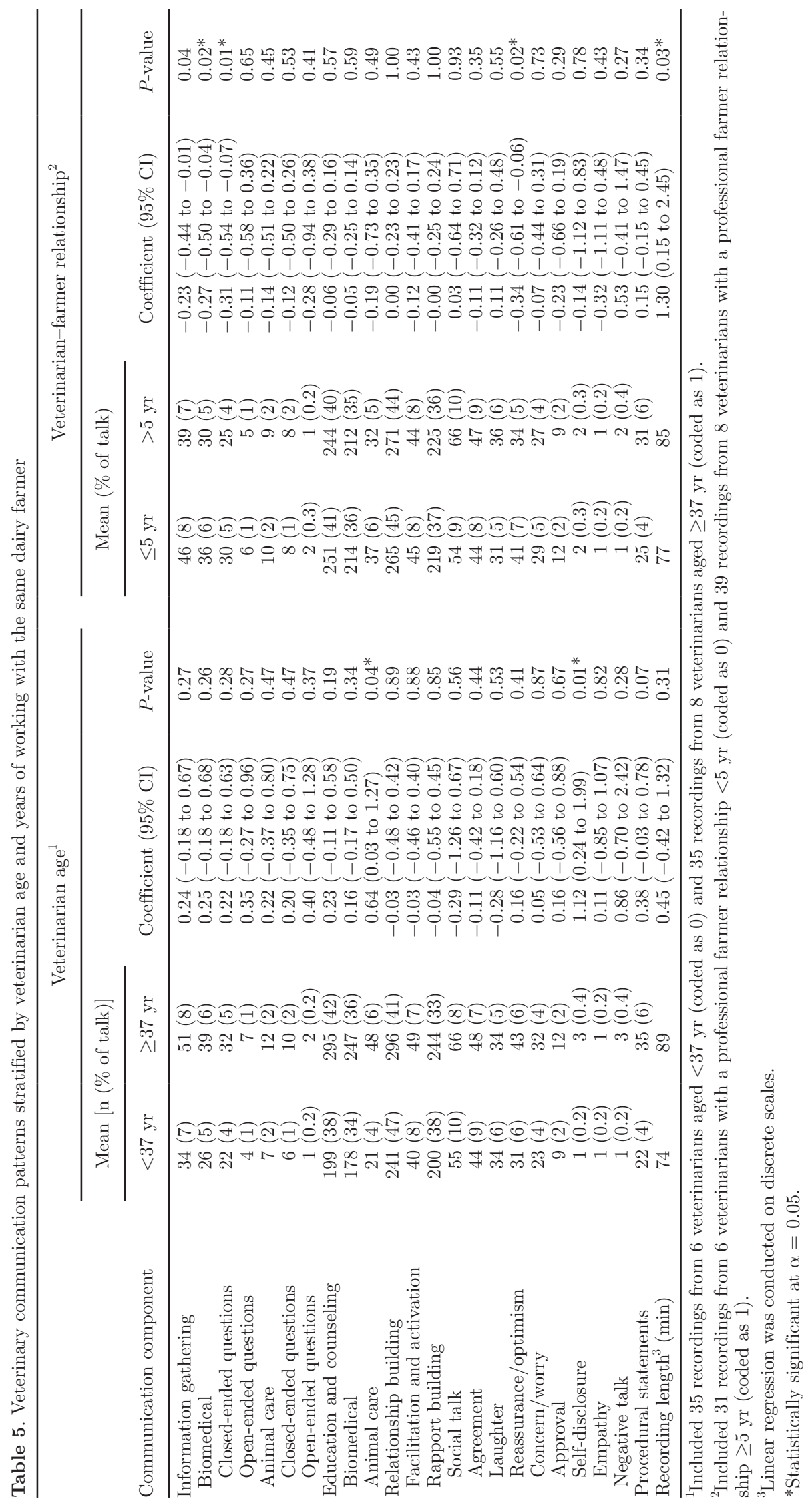


tice (Luby et al., 2013), veterinarian sex distribution in food animal practice (Jelinski and Barth, 2015), average milk production per cow (Ritter et al., 2015), and average number of lactating cows per farm (Canadian Dairy Information Centre, 2017). Although these comparisons supported the generalizability of the study, the size of the dairy farms participating in the study ranged from 34 to 500 lactating cows, and communication might be different on farms with substantially more cattle and where the labor is divided among more employees that are not necessarily involved in the decision-making process. Furthermore, veterinarians in this study were generally paid by the hour, which might lead to different communication than if they were paid per service.

Overall, distribution of veterinary talk among communication components was similar to results in companion animal medicine studies using the RIAS (Shaw et al., 2008; McArthur and Fitzgerald, 2013). However, dairy practitioners appeared to spend more talk on building a relationship compared with companion animal veterinarians $[44 \%$ of all veterinary talk compared with approximately 30\% (Shaw et al., 2008) and 26\% (McArthur and Fitzgerald, 2013), respectively]. Besides the longer visit time for dairy practitioners on the farm, which potentially allowed for more social talk, they generally had a long-lasting professional relationship with their clients and visited the farms on a regular basis, which likely further contributed to their increased use of social talk $(10 \%)$ compared with companion animal practitioners (1-3\%; Shaw et al., 2008; McArthur and Fitzgerald, 2013).

Gathering 3 to 7 recordings from each veterinarian decreased the risk of obtaining a nonrepresentative recording due to the observed high variability among farm visits. However, this approach resulted in a relatively small number $(\mathrm{n}=14)$ of participating veterinarians; therefore, potential differences between groups might not have been detected due to a lack of power. For example, the only difference between male and female veterinarians was the number of closed-ended animal care questions. However, when describing effects of sex dyads, on-farm interactions where farmer and veterinarian were both male had a higher RCC score than when at least 1 female was involved. This was consistent with the conclusion by Shaw et al. (2006) that opposite sexes increased the use of biomedical communication patterns. Similarly, in the human medical care context, sex-concordant visits were characterized by physicians

Table 6. Veterinary communication patterns stratified by whether they had received previous communication training

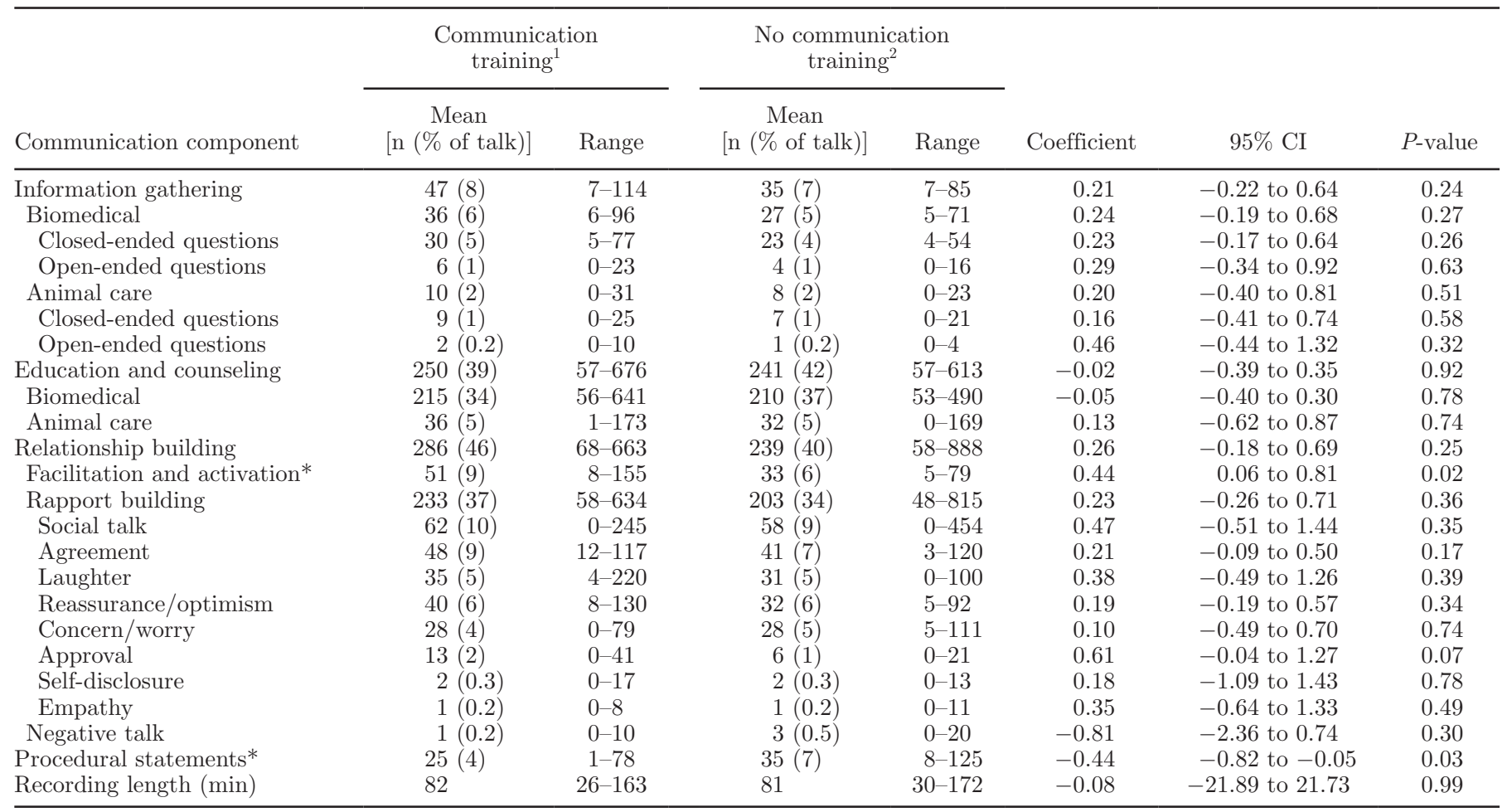

${ }^{1}$ Included 44 recordings from 8 veterinarians (coded as 1 ).

${ }^{2}$ Included 26 recordings from 6 veterinarians (coded as 0 ).

*Statistically significant at $\alpha=0.05$. 
Table 7. Comparison of communication patterns used by veterinarians during whole-herd conversations compared with discussions of individual animals

\begin{tabular}{|c|c|c|c|c|c|c|c|}
\hline \multirow[b]{2}{*}{ Communication component } & \multicolumn{2}{|c|}{ Herd $^{1}$} & \multicolumn{2}{|c|}{ Individual animal $^{2}$} & \multirow[b]{2}{*}{ Coefficient } & \multirow[b]{2}{*}{$95 \% \mathrm{CI}$} & \multirow[b]{2}{*}{$P$-value } \\
\hline & $\begin{array}{c}\text { Mean } \\
{[\mathrm{n}(\% \text { of talk })]}\end{array}$ & Range & $\begin{array}{c}\text { Mean } \\
{[\mathrm{n}(\% \text { of talk })]}\end{array}$ & Range & & & \\
\hline Information gathering* & $40(7)$ & $2-111$ & $5(5)$ & $0-24$ & -0.43 & -0.48 to 0.01 & 0.04 \\
\hline Biomedical & $31(6)$ & $2-86$ & $4(4)$ & $0-24$ & -0.12 & -0.38 to 0.14 & 0.37 \\
\hline Closed-ended questions & $26(5)$ & $1-69$ & $4(4)$ & $0-22$ & -0.06 & -0.04 to 0.21 & 0.66 \\
\hline Open-ended questions & $5(1)$ & $0-23$ & $1(1)$ & $0-5$ & -0.39 & -0.97 to 0.19 & 0.18 \\
\hline Open-ended questions & $1(0.2)$ & $0-10$ & $0.1(0.1)$ & $0-1$ & -0.73 & -1.89 to 0.44 & 0.22 \\
\hline Education and counseling* & $227(39)$ & $12-609$ & $44(50)$ & $0-141$ & 0.24 & 0.14 to 0.34 & $<0.001$ \\
\hline Biomedical* & $194(34)$ & $5-568$ & $42(48)$ & $0-121$ & 0.35 & 0.24 to 0.56 & $<0.001$ \\
\hline Animal care* & $33(5)$ & $0-173$ & $2(2)$ & $0-20$ & -0.93 & -1.35 to -0.52 & $<0.001$ \\
\hline Relationship building* & $255(45)$ & $30-885$ & $30(33)$ & $0-130$ & -0.25 & -0.35 to -0.14 & $<0.001$ \\
\hline Facilitation and activation & $42(8)$ & $4-146$ & $6(6)$ & $0-33$ & -0.11 & -0.36 to 0.14 & 0.41 \\
\hline Rapport building* & $211(36)$ & $16-812$ & $24(28)$ & $0-97$ & -0.26 & -0.39 to -0.14 & $<0.001$ \\
\hline Approval & $10(2)$ & $0-40$ & $1(1)$ & $0-6$ & -0.03 & -0.73 to 0.12 & 0.16 \\
\hline Self-disclosure & $2(0.3)$ & $0-17$ & $0.1(0.1)$ & $0-1$ & -0.69 & -1.94 to 0.57 & 0.28 \\
\hline Empathy* & $1(0.2)$ & $0-11$ & $0.4(0.4)$ & $0-5$ & 0.99 & 0.21 to 1.77 & 0.01 \\
\hline Negative talk & $2(0.3)$ & $0-20$ & $0.1(0.1)$ & $0-2$ & -0.29 & -1.33 to 0.75 & 0.58 \\
\hline Procedural statements & $26(5)$ & $1-125$ & $5(5)$ & $0-18$ & 0.04 & -0.24 to 0.33 & 0.77 \\
\hline
\end{tabular}

${ }^{1}$ Coded as 0

${ }^{2}$ Coded as 1 .

*Statistically significant at $\alpha=0.05$.

attempting to understand the whole person without focusing only on biomedical topics (Bertakis, 2009). Whereas biomedical communication was more likely in consultations with younger primary care physicians and older patients (Roter et al., 1997), age-dependent differences in dairy practitioners' communication patterns were limited in this study and not associated with RCC score.

Unlike previous research, where communication training was very variable among veterinarians (Shaw et al., 2006), here the difference between veterinarians who had $4 \mathrm{yr}$ of communication training during veterinary school and veterinarians who stated to have not received communication training was fairly distinct. One veterinarian stated that he or she received postgraduate communication education but because of the limited number of hours and lack of examination was classified as having not received communication training. Veterinarians with previous communication training used more statements related to farmer activation and facilitation. However, based on the overall similarity of communication patterns used by both groups, it appeared that either the communication training received was ineffective for the dairy context or the veterinarians had lost acquired skills after years in practice. Companion animal veterinarians with communication training were more likely to use a biomedical communication pattern (i.e., using a less relationship-centered approach) compared with veterinarians without communication training (Shaw et al., 2006), whereas the current study did not detect an effect on RCC score. However, Shaw et al. (2006) requested cautious interpretation of their study results due to the limited number of veterinarians who stated to have taken a communication course and the high variability of the duration, quality, and content and because most of the courses focused on practice management rather than communication skills training. Furthermore, other research demonstrated the effectiveness of communication training in human and companion animal medicine (e.g., Latham and Morris, 2007; Shaw et al., 2010; Artemiou et al., 2013; Clayton et al., 2013). More research is needed to assess the benefits of communication education over time, especially in the farm animal context.

The most differences in communication patterns used were revealed when comparing conversations related to the whole herd compared with issues regarding an individual animal. Whole-herd discussions included more social talk and laughter, similar to results by Shaw et al. (2008), who compared veterinary practice wellness appointments with visits where a companion animal presented with a health problem. Also similar to results 
Table 8. Univariable associations of demographic variables with relationship-centered care (RCC) score

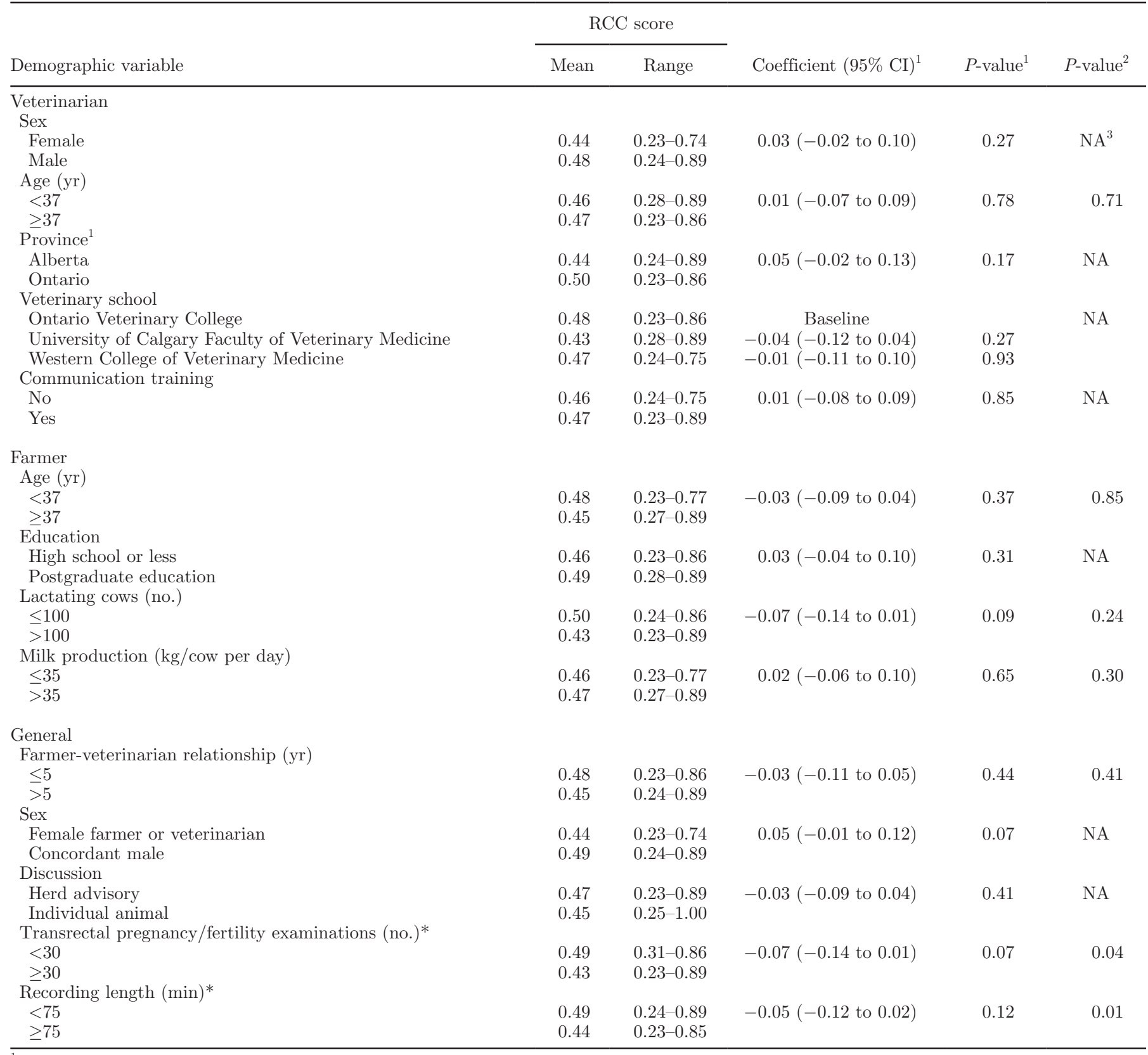

${ }^{1}$ Based on linear regression analysis with stratified explanatory variables.

${ }^{2}$ Based on linear regression analysis with explanatory variables on a discrete scale.

${ }^{3}$ Not applicable.

*Statistically significant at $\alpha=0.05$.

Table 9. Multivariable associations of demographic variables on relationship-centeredness care score

\begin{tabular}{lccc}
\hline Demographic variable & Coefficient & $95 \%$ CI & $P$-value \\
\hline Province Ontario & 0.09 & 0.02 to 0.15 & 0.01 \\
Concordant male $_{\text {Milk production }}^{1}(\mathrm{~kg} / \mathrm{cow}$ per day) & 0.08 & 0.02 to 0.14 & 0.01 \\
Recording length $^{1}(\mathrm{~min})$ & 0.01 & 0.00 to 0.02 & 0.02 \\
\hline
\end{tabular}

${ }^{1}$ Based on linear regression analysis with explanatory variables on a discrete scale (where applicable). 
from Shaw et al. (2008), discussions revolving around an individual animal contained more statements of concern and biomedical talk.

Patient-centeredness is generally regarded as an important component of effective medical care (Weiner et al., 2013). However, lack of agreement regarding definition and measure of patient-centeredness (or "relationship-centeredness" in veterinary medicine) makes it difficult to compare evaluations that used different analytical tools (Mead and Bower, 2000; Mole et al., 2016). Stewart et al. (2003) identified 6 interconnecting components that contribute to the patient-centeredness of the medical encounter: (1) understanding the whole person, (2) exploring both the disease and the illness experience, (3) enhancing the doctor-patient relationship, (4) finding common ground regarding management, (5) incorporating disease prevention and health promotion, and (6) being realistic about personal limitations and barriers (e.g., availability of time and resources). Although the veterinary context might be more comparable with pediatric consultations, in which the primary caretakers are not the patient but rather the decision makers, the general principles of patientor relationship-centeredness are likely transferable, and a subset of the 6 components has been applied to assess euthanasia discussions in companion animal medicine (Nogueira Borden et al., 2010; Williams and Jewell, 2012). In this study, being the first to calculate the relationship-centeredness of dairy farm interactions using the RIAS, variables that enable a good relationship, veterinarian's interest in the farm management as a whole, shared power and decision-making were contrasted against variables representing veterinary dominance and a sole focus on medical issues. Overall, in this study, veterinarians' RCC score had a mean of 0.47, which was lower than the RIAS-based RCC score (mean: 1.0) reported in companion animal interactions (Kanji et al., 2012) but similar to the RCC score when only appointments with a biomedical communication pattern were taken into account (0.40; Shaw et al., 2006). Although no gold standard is established for the relationship-centeredness of medical interactions in the veterinary context, this relatively low RCC score in combination with low use of other tools that have been demonstrated to improve medical outcomes (e.g., open-ended questions to assess farmers' views and empathy statements; Roter and Hall, 1987; McArthur and Fitzgerald, 2013) might constitute an opportunity to enhance veterinary on-farm communication.

Because statements related to cows' pregnancy or fertility status were coded as biomedical information giving, number of transrectal palpations was counted by RIAS coders. This was done to assess the influence of the number of pregnancy or fertility assessments on
RCC score. In the univariable analysis, there was a negative relationship between number of pregnancy or fertility assessments and RCC score, perhaps due to inflation of the denominator of the RCC. Although this relationship was not confirmed in the multivariable analysis, length of recordings, which was highly correlated with number of pregnancy or fertility assessments, had a negative relationship with RCC score. One could assume that $\mathrm{HH} \& \mathrm{PM}$ visits with more transrectal assessments took longer and contained more biomedical information-giving statements related to pregnancy or fertility diagnosis, but because of multicollinearity in the multivariable model, only length of recording remained significant.

A limitation to consider is the potential for selection bias. Although the authors believe that a comprehensive sampling frame was created between the Alberta Johne's Disease Initiative database and their familiarity with dairy practitioners in Alberta, it is possible that eligible veterinarians were not included. Differences between veterinarians from Alberta and Ontario could have been due to different participant selection methods. Whereas veterinarians in Alberta at a feasible driving distance from Calgary were invited to participate in the study, in Ontario, veterinarians who enrolled in a Dairy Health Management Continuing Education Program were asked to volunteer. That veterinarians selected the farmers for recording could be another source of bias because veterinarians might have selected only farmers with whom they get along well. Although it is difficult to assess exactly how nonrandom selection of farmers influenced veterinarians' communication, it can, for example, be assumed that veterinarians use more social talk with farmers with whom they feel more comfortable. In contrast, awareness of being recorded could have altered participant behavior and reduced social talk, as participants focused on the professional aspects of the HH\&PM visit (Derks et al., 2013; Ritter et al., 2018). However, compared with having a researcher present, providing veterinarians with action cameras was likely less disturbing for the course of the farm visit and exhibited communication patterns.

Veterinarians could have made an effort to include communication tools they thought would be evaluated positively, and therefore recorded interactions might have been an overestimation of veterinarians' communication skills. If so, presumably veterinarians with previous communication training would be more likely to recall these skills, although communication was very similar between veterinarians with and without training.

This article reports on veterinary communication during HH\&PM visits in detail and describes their association with demographic factors, enabling compari- 
sons with other medical contexts and providing a first reference from the dairy environment. To evaluate the quality of the advisory from the farmer's perspective, the next logical step would be to correlate communication to outcomes such as farmer satisfaction or adherence.

\section{CONCLUSIONS}

Recording of dairy HH\&PM farm visits identified distinct veterinary communication patterns and their associations with demographic variables. Therefore, this study provided the dairy farm counterpart to studies conducted in companion animal medicine more than a decade ago. Identification of opportunities to enhance veterinary communication skills in a dairy setting is a first step to inform and improve veterinary communication education.

\section{ACKNOWLEDGMENTS}

This study was funded by the Margaret Gunn Endowment for Animal Research, the Izaak Walton Killam Trust, and Canada's Natural Sciences and Engineering Research Council Industrial Research Chair Program, with industry contributions from Alberta Milk (Edmonton, AB, Canada), Dairy Farmers of Canada (Ottawa, ON, Canada), Dairy Farmers of Manitoba (Winnipeg, MB, Canada), British Columbia Dairy Association (Burnaby, BC, Canada), Westgen Endowment Fund (Milner, BC, Canada), Canadian Dairy Network (Guelph, ON, Canada), and CanWest DHI (Guelph, ON, Canada). The authors thank John Kastelic for editing the manuscript.

\section{REFERENCES}

Adams, C. L., and S. Kurtz. 2017. Skills for Communicating in Veterinary Medicine. 1st ed. MHRI, Parsippany, NJ.

Artemiou, E., C. L. Adams, A. Vallevand, C. Violato, and K. G. Hecker. 2013. Measuring the effectiveness of small-group and web-based training methods in teaching clinical communication: A case comparison study. J. Vet. Med. Educ. 40:242-251.

Bard, A. M., D. C. Main, A. M. Haase, H. R. Whay, E. J. Roe, and K. K. Reyher. 2017. The future of veterinary communication: Partnership or persuasion? A qualitative investigation of veterinary communication in the pursuit of client behaviour change. PLoS One 12:e0171380.

Bertakis, K. D. 2009. The influence of gender on the doctor-patient interaction. Patient Educ. Couns. 76:356-360.

Canadian Dairy Information Centre. 2017. Dairy facts and figures. Accessed May 11, 2018. www.dairyinfo.gc.ca.

Cipolla, M., and A. Zecconi. 2015. Short communication: Study on veterinarian communication skills preferred and perceived by dairy farmers. Res. Vet. Sci. 99:60-62.

Clayton, J. M., P. N. Butow, A. Waters, R. C. Laidsaar-Powell, A. O'Brien, F. Boyle, A. L. Back, R. M. Arnold, J. A. Tulsky, and M. H. Tattersall. 2013. Evaluation of a novel individualised commu- nication-skills training intervention to improve doctors' confidence and skills in end-of-life communication. Palliat. Med. 27:236-243.

Coe, J. B., C. L. Adams, and B. N. Bonnett. 2009. Prevalence and nature of cost discussions during clinical appointments in companion animal practice. J. Am. Vet. Med. Assoc. 234:1418-1424.

Derks, M., B. van Woudenbergh, M. Boender, W. Kremer, T. van Werven, and H. Hogeveen. 2013. Veterinarian awareness of farmer goals and attitudes to herd health management in The Netherlands. Vet. J. 198:224-228.

Jansen, J., H. Klinkert, R. J. Renes, and T. J. G. M. Lam. 2010. Effective communication of veterinary advice: Interaction between the veterinarian and the farmer. Pages 185-191 in Mastitis Research into Practice: Proceedings of the 5th IDF Mastitis Conference. J. E. Hillerton, ed. Vetlearn, Christchurch, New Zealand.

Jelinski, M. D., and K. K. Barth. 2015. Survey of western Canadian veterinary practices: A demographic profile. Can. Vet. J. 56:12451251.

Kanji, N., J. B. Coe, C. L. Adams, and J. R. Shaw. 2012. Effect of veterinarian-client-patient interactions on client adherence to dentistry and surgery recommendations in companion-animal practice. J. Am. Vet. Med. Assoc. 240:427-436.

Lam, T. J. G. M., J. Jansen, B. H. P. Van den Borne, R. J. Renes, and H. Hogeveen. 2011. What veterinarians need to know about communication to optimise their role as advisor on udder health in dairy herds. N. Z. Vet. J. 59:8-15.

Latham, C. E., and A. Morris. 2007. Effects of formal training in communication skills on the ability of veterinary students to communicate with clients. Vet. Rec. 160:181-186.

Luby, C. D., K. McIntyre, and M. D. Jelinski. 2013. Skills required of dairy veterinarians in western Canada: A survey of practicing veterinarians. Can. Vet. J. 54:267-270.

McArthur, M. L., and J. R. Fitzgerald. 2013. Companion animal veterinarians' use of clinical communication skills. Aust. Vet. J. 91:374-380.

McDermott, M. P., V. A. Tischler, M. A. Cobb, I. J. Robbe, and R. S. Dean. 2015. Veterinarian-client communication skills: Current state, relevance, and opportunities for improvement. J. Vet. Med. Educ. 42:305-314.

Mead, N., and P. Bower. 2000. Patient-centredness: A conceptual framework and review of the empirical literature. Soc. Sci. Med. 51:1087-1110.

Mee, J. F. 2007. The role of the veterinarian in bovine fertility management on modern dairy farms. Theriogenology 68(Suppl. 1):S257-S265.

Mellanby, R. J., S. M. Rhind, C. Bell, D. J. Shaw, J. Gifford, D. Fennell, C. Manser, D. P. Spratt, M. J. Wright, S. Zago, and N. P. Hudson. 2011. Perceptions of clients and veterinarians on what attributes constitute "a good vet." Vet. Rec. 168:616.

Mole, T. B., H. Begum, N. Cooper-Moss, R. Wheelhouse, P. MacKeith, T. Sanders, and V. Wass. 2016. Limits of "patient-centredness": Valuing contextually specific communication patterns. Med. Educ. 50:359-369.

Mossop, L., C. Gray, A. Blaxter, A. Gardiner, K. MacEachern, P. Watson, K. Whittlestone, and I. Robbe. 2015. Communication skills training: What the vet schools are doing. Vet. Rec. 176:114-117.

Nogueira Borden, L. J., A. C. Bonnett, B. N. Shaw Jr., and C. S. Ribble. 2010. Use of the measure of patient-centered communication to analyze euthanasia discussions in companion animal practice. J. Am. Vet. Med. Assoc. 237:1275-1287.

Pires, C. M., and A. M. Cavaco. 2014. Communication between health professionals and patients: Review of studies using the RIAS (Roter Interaction Analysis System) method. Rev. Assoc. Med. Bras. 60:156-172.

Ritter, C., H. W. Barkema, and C. L. Adams. 2018. Action cameras and the Roter interaction analysis system to assess veterinarianproducer interactions in a dairy setting. Vet. Rec. 182:227.

Ritter, C., G. P. Kwong, R. Wolf, C. Pickel, M. Slomp, J. Flaig, S. Mason, C. L. Adams, D. F. Kelton, J. Jansen, J. De Buck, and H. W. Barkema. 2015. Factors associated with participation of Alberta dairy farmers in a voluntary, management-based Johne's disease control program. J. Dairy Sci. 98:7831-7845. 
Roter, D., and S. Larson. 2002. The Roter interaction analysis system (RIAS): Utility and flexibility for analysis of medical interactions. Patient Educ. Couns. 46:243-251.

Roter, D. L., M. Stewart, S. M. Putnam, M. Lipkin Jr., W. Stiles, and T. S. Inui. 1997. Communication patterns of primary care physicians. J. Am. Vet. Med. Assoc. 277:350-356.

Roter, D. L., and J. A. Hall. 1987. Physician's interviewing styles and medical information obtained from patients. J. Gen. Intern. Med. $2: 325-329$.

Shaw, J. R., C. L. Adams, B. N. Bonnett, S. Larson, and D. L. Roter. 2004. Use of the Roter interaction analysis system to analyze veterinarian-client-patient communication in companion animal practice. J. Am. Vet. Med. Assoc. 225:222-229.

Shaw, J. R., C. L. Adams, B. N. Bonnett, S. Larson, and D. L. Roter. 2008. Veterinarian-client-patient communication during wellness appointments versus appointments related to a health problem in companion animal practice. J. Am. Vet. Med. Assoc. 233:15761586 .

Shaw, J. R., G. E. Barley, A. E. Hill, S. Larson, and D. L. Roter. 2010. Communication skills education onsite in a veterinary practice. Patient Educ. Couns. 80:337-344.

Shaw, J. R., B. N. Bonnett, C. L. Adams, and D. L. Roter. 2006 Veterinarian-client-patient communication patterns used during clinical appointments in companion animal practice. J. Am. Vet. Med. Assoc. 228:714-721.

Silverman, J., S. Kurtz, and J. Draper. 2013. Skills for Communicating with Patients. 3rd ed. Radcliffe, London, UK.

Stewart, M., J. B. Brown, W. W. Weston, I. R. McWhinney, C. L. McWilliam, and T. R. Freeman. 2003. Patient-Centered Medicine: Transforming the Clinical Method. 2nd ed. Radcliffe, London, UK. Weiner, S. J., A. Schwartz, K. Cyrus, A. Binns-Calvey, F. M. Weaver, G. Sharma, and R. Yudkowsky. 2013. Unannounced standardized patient assessment of the Roter interaction analysis system: The challenge of measuring patient-centered communication. J. Gen. Intern. Med. 28:254-260.

Williams, D., and J. Jewell. 2012. Family-centred veterinary medicine: Learning from human paediatric care. Vet. Rec. 170:79-80.

Winder, C. B., S. J. LeBlanc, D. B. Haley, K. D. Lissemore, M. A Godkin, and T. F. Duffield. 2016. Practices for the disbudding and dehorning of dairy calves by veterinarians and dairy producers in Ontario, Canada. J. Dairy Sci. 99:10161-10173.

Wolf, R., H. W. Barkema, J. De Buck, and K. Orsel. 2015. Factors affecting management changes on farms participating in a Johne's disease control program. J. Dairy Sci. 98:7784-7796. 